\title{
关于可积 $\mathfrak{G}(\boldsymbol{A})$ 模权集的注记
}

\author{
张知学
}

(河北大学数学系, 保定 071002)

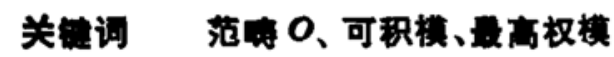

本文采用文献[1]中的符号和术语. 设 $A$ 为一个 $n \times n$ 广义 Cartan 矩阵, $\mathscr{G}(A)$ 为络 合于 $A$ 的 Kac-Moody 代数, $\mathfrak{H}$ 为其 Cartan 子代数.

$$
\Pi-\left\{\alpha_{1}, \cdots, \alpha_{n}\right\} . \Pi^{v}-\left\{\alpha_{1}^{v}, \cdots, \alpha_{n}^{y}\right\}
$$

分别为根基和对偶根基. 令 $\Delta, Q$ 和 $P$ 分别表示 $\mathcal{G}(A)$ 的根系、根格和权格, 而 $\Delta_{+}$为正根 系, $Q_{+}-\sum_{i=1}^{n} \mathrm{Z}_{+} \alpha_{i}, P_{+}$是支配整权的集合. 设 $e_{i}, f_{i}(i-1,2, \cdots, n)$ 为 $\mathfrak{B}(A)$ 的 - 组 Chevally 基, 使 $\left[e_{i}, f_{i}\right]-\alpha_{i}^{V}$. 又设

$$
\mathfrak{B}(\boldsymbol{A})-\mathfrak{N}_{+} \oplus \mathfrak{S} \oplus \mathfrak{N}_{-}
$$

是 $\Phi(A)$ 关于 $\mathfrak{S}$ 的三角分解. $\mathscr{S}(A)$ 模 $V$ 称为范畴 $O$ 的,如果

$1^{\circ} V$ 是 S-可对角化的, 即 $V-\bigoplus_{i \in \mathbb{S}^{-}} V_{2}$,

$2^{\circ} \operatorname{dim} V_{\lambda}<\infty, \forall \lambda \in \mathfrak{H}^{*}$,

$3^{\circ}$ 存在有限个元素 $\lambda_{1}, \cdots, \lambda_{s} \in \mathfrak{S}^{*}$, 使 $V$ 的权集 $P(V)$ 满足

$$
P(V) \subset \bigcup_{i=1}^{i} D\left(\lambda_{i}\right) \text {, }
$$

其中 $D\left(\lambda_{i}\right)-\left\{\mu \in \mathfrak{S}^{*} \mid \mu \leqslant \lambda_{i}\right\}(i-1,2, \cdots, s)$.

范畴 $O$ 模的重要例子是最高权模. 一个最高权模的最高权 $\Lambda$ 和一个最高权向量 $\nu_{+}$可以 简单地说成最高权对 $\left(\Lambda, v_{+}\right)$.

$\mathcal{B}(A)$ 模 $V$ 称为可积的, 如果 $V$ 是 $\mathfrak{S}$-可对角化的, 并且所有 $e_{i}, f_{i}(i-1,2, \cdots, n)$ 都局 部第零地作用于 $V$. 为了后面的需要, 先介绍三个引理。

引理 1 设 $V$ 是一个最高权 $\mathscr{B}(A)$ 模, 具有最高权对 $\left(\Lambda, v_{+}\right)$则下列诸条件等价:

$1^{\circ} \mathrm{V}$ 是可积的;

$2^{\circ}$ 对任意 $i, 1 \leqslant i \leqslant n$, 存在正整数 $n_{i}$, 使

$3^{\circ} \Lambda \in P_{+}$, 且对任意 $i, 1 \leqslant i \leqslant n$,

$$
f_{i}^{n} i v_{+}-0 \text {; }
$$

$$
f^{\left(A, a_{i}^{p}\right)+1} \nu_{+}-0
$$

1991-10-24收稍, 1992-04-24 收悠改稆 
证 文献[1]中 10.1 已证明 $1^{\circ} \Longleftrightarrow 2^{\circ}$. 现只需证 $2^{\circ} \Rightarrow 3^{\circ}$. 设 $n_{i}$ 是便

$$
f_{i}^{n} i v_{+}=0
$$

成立的最小正整数. 由于

$$
0-e_{i} f_{i}^{n_{i} \nu_{+}}-n_{i}\left(\left\langle\Lambda, \alpha_{i}^{\nu}\right\rangle-n_{i}+1\right) f_{i}^{n_{i}-1} \cdot \nu_{+},
$$

可知 $n_{i}-\left\langle\Lambda, \alpha_{i}^{V}\right\rangle+1$, 并由此可知 $\left\langle\Lambda, \alpha_{i}^{V}\right\rangle \geqslant 0$, 即 $\Lambda \in P_{+}$.

引理 2 设 $V$ 是一个以 $\Lambda \in \mathfrak{S}^{*}$ 为最高权的最高权模, 则 对任意 $\lambda \in P(V), \lambda \neq \Lambda$, 存在 $\alpha_{i} \in \Pi$, 使 $\lambda+\alpha_{i} \in P(V)$.

证 每个 $\lambda \in P(V)$ 都可表为 $\lambda-\Lambda-\sum_{i=1}^{n} k_{i} \alpha_{i}, k_{i} \geqslant 0$.

引理 3 设 $V$ 是范畴 $O$ 的 $B(A)$ 模. 则对任意 $\lambda \in P(V)$, 存在 $P(V)$ 中的极大元

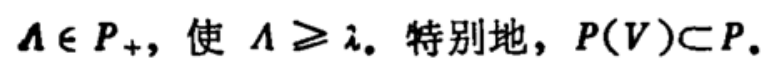

证 令 $P_{2}-\{\mu \in P(V) \mid \mu \geqslant \lambda\}$. 由范畴 $O$ 的定义, $P_{2}-\bigcup_{i=1}^{1}\left(D\left(\lambda_{i}\right) \cap P_{2}\right)$. 若 $\lambda \in D\left(\lambda_{i}\right)$, 则 $D\left(\lambda_{i}\right) \cap P_{1}-\left\{\lambda_{i}-\varphi \mid \lambda_{i}-\varphi \geqslant \lambda\right\}$ 是有限集; 若 $\lambda \in D\left(\lambda_{i}\right)$, 则 $D\left(\lambda_{i}\right) \cap P_{2}$ 是 空集,因而 $P_{2}$ 为有限集, 从而 $P_{\lambda}$ 中有极大元 $\Lambda$. 显然, $\Lambda$ 也是 $P(V)$ 中的极大元.

取 $v \in V_{\Delta}, v \neq 0$. 由于

$$
\mathfrak{N}_{+} \cdot \nu \subset \sum_{a \in O_{+}} V_{\lambda+a}-0
$$

可知

$$
V_{1}:=U(\bigoplus(A)) \cdot v
$$

是一个最高权模, 具有最高权对 $(\Lambda, v)$. 作为 $V$ 的子模, $V_{1}$ 是可积的. 由引理 $1, \Lambda \in P_{+}$. 又 因 $\lambda \leqslant \Lambda$, 即存在 $a \in Q_{+}$, 使 $\lambda-\Lambda-\alpha$, 所以 $\lambda \in P$.

下面讨论可积最高权 $\mathscr{B}(A)$ 模权集的某些性质.

定理 1 设 $V_{1}$ 和 $V_{2}$ 是两个以 $\Lambda \in P_{+}$为最高权的可积最高权 $\mathscr{G}(A)$ 模, 则它们的权 集相同, 即 $P\left(V_{1}\right)-P\left(V_{2}\right)$.

证设 $n$ 为非负整数, 令

$$
P_{n}\left(V_{i}\right)-\left\{\lambda \in P\left(V_{i}\right) \mid h r(\Lambda-\lambda)-n\right\}, i-1,2,
$$

注意 $P_{0}\left(V_{2}\right)-P_{0}\left(V_{2}\right)-\{\Lambda\}$, 当 $n>0$ 时, 可能 $P_{n}\left(V_{i}\right)-\phi$, 对 $n$ 用归纳法证明

$$
P_{*}\left(V_{1}\right)-P_{*}\left(V_{2}\right) \text {. }
$$

假定对一切 $k<n$, 有 $P_{k}\left(V_{1}\right)-P_{k}\left(V_{2}\right)$. 若 $P_{n}\left(V_{1}\right) \neq \phi$, 设 $\lambda \in P_{*}\left(V_{1}\right)$, 由引理 2 存 在 $\alpha_{i} \in \Pi$, 使 $\lambda+\alpha_{i} \in P\left(V_{1}\right)$. 考虑 $P\left(V_{1}\right)$ 中通过权 $\lambda$ 的 $\alpha_{i}$ 权链

$$
\lambda-p \alpha_{i}, \cdots, \lambda, \cdots, \lambda+q i x_{i},
$$

显然 $q \geqslant 1$, 并且

$$
\left\langle\lambda+q \alpha_{i}, \alpha_{i}^{p}\right\rangle-\left\langle\lambda, \alpha_{i}^{v}\right\rangle+2 q-p-q+2 q-p+q \geqslant q .
$$

由于 $\lambda+q \alpha_{i} \in P_{n-q}\left(V_{1}\right)$ 和 $q \geqslant 1$, 由归纳假设

$$
\lambda+q \alpha_{i} \in P_{n-q}\left(V_{2}\right) \text {. }
$$

考虑 $P\left(V_{2}\right)$ 中通过权 $\lambda+q \alpha_{i}$ 的 $\alpha_{i}$ 权链

$$
\left(\lambda+q \alpha_{i}\right)-S \alpha_{i}, \cdots, \lambda+q \alpha_{i}, \cdots,\left(\lambda+q \alpha_{i}\right)+t \alpha_{i},
$$

因 $s-i-\left\langle\lambda+q \alpha_{i}, \alpha_{i}^{\nu}\right\rangle \geqslant q$, 从而 $s \geqslant q$. 这表明 $\lambda$ 包含在权链 $(*)$ 中, 即 $\lambda \in P_{n}\left(V_{2}\right)$. 这 样就证明了 $P_{n}\left(V_{1}\right)-P_{*}\left(V_{2}\right)$, 并且显然 $P_{*}\left(V_{1}\right)-\phi$ 当且仅当 $P_{*}\left(V_{2}\right)-\phi$. 
由定理 1, 可积最高权模的权集是由其最高权 $\Lambda\left(\Lambda \in P_{+}\right)$完全确定的. 我们把这个权 果记作 $P(\Lambda)$.

我们知道, Verma 模 $M(\Lambda)\left(\Lambda \in \mathscr{S}^{*}\right)$ 是具有泛性的最高权模, 即任一个以 $\Lambda$ 为最高权 的最高权模都是 $M(\Lambda)$ 的同态象. 在可积模范畴中也有具有这种泛性的模. 这就是下面的 定义 1 和定理 2 .

定义 1 设 $\Lambda \in P_{+} . M_{I}(\Lambda)$ 是一个以 $\Lambda$ 为最高权的可积最高权 $\mathscr{G}(A)$ 模. $M_{I}(\Lambda)$ 称为可积 Verma 模, 如果任何一个以 $\Lambda$ 为最高权的可积最高权模都是 $M_{I}(\Lambda)$ 的同态象.

定理 2 对 $\Lambda \in P_{+}$, 存在唯一可积 Verma 模 $M_{I}(\Lambda)$.

证设 $M(\Lambda)$ 是 Verma 模, 具有最高权对 $\left(\Lambda, \tilde{v}_{+}\right)$. 令

$$
v_{i}-f_{i}^{\left(\Lambda, \alpha_{i}^{V}\right)+1} \tilde{v}_{+}, 1 \leqslant i \leqslant n,
$$

则 $v_{i}$ 是 $M(\Lambda)$ 中的极大向量. 事实上, 由文献[1]的 (3.2.4) 式可知 $e_{i} v_{i}-0$, 而当 $i \neq i$ 时, $e_{i} v_{i}-f_{i}^{\left(\Lambda, \omega_{i}^{V}\right)+1} e_{i} \tilde{v}_{+}-0$. 现令

$$
M_{1}-\sum_{i=1}^{n} U\left(\Re_{-}\right) \cdot v_{i},
$$

显然 $M_{1}$ 是 $M(\Lambda)$ 的真子模. 再令

$$
M_{l}(\Lambda)-M(\Lambda) / M_{1}, v_{+}-\tilde{v}_{+}+M_{1},
$$

则 $M_{1}(\Lambda)$ 是具有最高权对 $\left(\Lambda, v_{+}\right)$的最高权模. 由引理 $1\left(3^{\circ}\right), M_{1}(\Lambda)$ 是可积的.

设 $V$ 是任一可积最高权模, 具有最高权对 $\left(\Lambda, v_{+}^{\prime}\right)$. 由 Verma 模 $M(\Lambda)$ 的泛性可知存 在满同态映射 $\varphi: M(\Lambda) \rightarrow V$, 使 $\varphi\left(\tilde{v}_{+}\right)-v_{+}^{\prime}$. 因 $V$ 是可积的, 由引理 1 知,

$$
\varphi\left(\nu_{i}\right)-f_{i}^{\left(A+x_{i}^{\prime}\right)+1} v_{+}^{\prime}-0,
$$

从而 $M_{1} \subset \mathrm{Ker} \varphi$. 根据同态分解定理, 存在唯一同态 $\varphi_{*}: M_{l}(\Lambda) \rightarrow V$ 使 $\varphi-\varphi_{*}{ }^{\circ}$, 其中 $\pi$ 是 $M(\Lambda)$ 到 $M_{I}(\Lambda)$ 的自然同态,并且 $p_{*}\left(\nu_{+}\right)-v_{+}^{\prime}$.

由定理 1 和 2 可得如下

推论设 $N$ 是可积 Verma 模 $M_{l}(\Lambda)$ 的任一真子模,则对任意 $\lambda \in P(\Lambda)$,

$$
\operatorname{dim}\left(M_{I}(\Lambda)\right)_{2}>\operatorname{dim} N_{2} \text {. }
$$

证 令 $V-M_{I}(\Lambda) / N$, 则 $V$ 是以 $\Lambda$ 为最高权的可积最高权模, 它与 $M_{I}(\Lambda)$ 有相同 的权集 $P(\Lambda)$, 从而 $\lambda$ 也是 $V$ 的权.

必须注意,由于 Verma 模 $M(\Lambda)$ 是秩 1 的自由 $U\left(\Re_{-}\right)$模, 由引理 1 , Verma 模不是可 积的,因而可积 Verma 模 $M_{I}(\Lambda)$ 并不是 Verma 模.

对于给定的 $\Lambda \in P_{+}$, 设 $N(\Lambda)$ 是可积 Verma 模 $M_{I}(\Lambda)$ 的极大真子模, 则

$$
L(\Lambda):=M_{1}(\Lambda) / N(\Lambda)
$$

是不可约最高权模, 当然是可积模. 因此, 在以 $\Lambda \in P_{+}$为最高权的可积最高权模中, $M_{I}(\Lambda)$ 和 $L(\Lambda)$ 可以看做是“最大”的和“最小”的. 当然, 当矩阵 $A$ 是可对称化时, 所有这些可积最 高权模是同构的(文献 [1],推论 10.4).

下面从饱和权集的角度来刻划权集 $P(\Lambda)$.

定义 2 权格 $P$ 中一个子集 $S$ 称为饱和的, 如果对任意 $\lambda \in S, \alpha \in \Delta^{\infty}$ 和 0 与 $\left\langle\lambda, \alpha^{\circ}\right\rangle$ 之间的任意整数 $k$, 都有 $\lambda-k \alpha \in S$.

设 $\Lambda \in P_{+}$, 由引理 $3 P(\Lambda) \subset P$. 另一方面, 由可积最高权模过权 $\lambda$ 的 $\alpha$ 权链 $\left(\alpha \in \Delta^{\infty}\right)$ 
可知, $P(\Lambda)$ 是 $P$ 中的饱和集 (见文献 [1]命题 11.1) 考虑权格 $P$ 中所有包含 $\Lambda$ 的饱和集之交 $S_{\Lambda}$. 显然 $S_{\Lambda}$ 仍是饱和集, 它是包含 $\Lambda$ 的最小饱和集. 由于权集 $P(\Lambda)$ 也是包含 $\Lambda$ 的饱和 集,所以 $S_{A} \subset P(\Lambda)$. 进一步可以证明

\section{定理 $3 S_{A}-P(\Lambda)$.}

证 若 $S_{A} \neq P(\Lambda)$, 取 $\lambda \in P(\Lambda) \backslash S_{A}$, 使 $h t(\Lambda-\lambda)$ 极小. 由引理 2, 存在 $\alpha_{i} \in \Pi$, 使 $\lambda+\alpha_{i} \in P(\Lambda)$. 考虑 $P(\Lambda)$ 中通过权 $\lambda$ 的 $\alpha_{i}$ 权链

$$
\lambda-p \alpha_{i}, \cdots, \lambda, \cdots, \lambda+q \alpha_{i},
$$

则 $\left\langle\lambda, \alpha_{i}^{p}\right\rangle-p-q, q \geqslant 1$. 因 $h t(\Lambda-\lambda)>h t\left(\Lambda-\left(\lambda+q \alpha_{i}\right)\right)$, 由 $h t(\Lambda-\lambda)$ 的极小 性知 $\lambda+q \alpha_{i} \in S_{A \text {. 但是 }}$

$$
\left\langle\lambda+q \alpha_{i}, \alpha_{i}^{\nu}\right\rangle-p-q+2 q-p+q \geqslant q \geqslant 1,
$$

从而对任意整数 $k, 0 \leqslant k \leqslant p+q$, 有

$$
i+q \alpha_{i}-k \alpha_{i} \in S_{\Delta \bullet}
$$

显然, $k$ 可以取到 $q$, 从而 $\lambda \in S_{A}$, 矛盾.

\section{今 考文䘞}

[1] Kac, V. G., Infinite Dimensional Lie Algebras, Cambridge Univeraity Press, 1985. 